\title{
An Approach of Risk Management for Multimedia Streaming Service in Cloud Computing
}

\author{
Youjin Song $^{1}$ and Yasheng Pang ${ }^{2}$ \\ ${ }^{1,2}$ Department of Information Management, Dongguk University \\ ${ }^{1}$ song@dongguk.ac.kr, ${ }^{2}$ pangpang7117@gmail.com
}

\begin{abstract}
Along with internet broadcasting technology, cloud broadcasting is gradually becoming the development tendency to audience view. But the problems which extsted in cloud environment also may affect in cloud based multimedia streaming service orea. In this paper, we proposes an approach of risk management for multimedia streaming service in cloud computing. Through previous study, we recommended a leveraged BMIS model for cloud risk management. To apply this model into cloud broadcasting environment, considerate with the new feature required in multimedia streaming service, Cwe found the significance of performance in cloud based streaming service. Then we integrate and leverage the new feature to the remodeled BMIS model, and finally develop a performance targeted leveraged BMIS model for cloud multimedia streaming risk management.
\end{abstract}

Keywords: Multimedia streaming, Cl@ud computing, risk management, BMIS, Performance

\section{Introduction}

Traditional multimedia broadcasters use the physical TV network to distribute their programs, there ane occasional service interrupt or breakdown during the broadcasting. Nowadays with the development of information technology, internet TV, IPTV, Mobile TV etc. racing to emerge in audience's view. Cloud based multimedia streaming service already moved on the agenda. Through cloud computing, broadcasters can hold down their costs by using the infrastructure and software service provided by the cloud providers, and more convenient accéss to the latest technical advances. For audiences, cloud based multimedia streaming service can offer a more interactive and higher definition image transmission.

But the problems here are more severe and various in cloud environment. Issues like users' privacy service attack and more kinds of potential problems lurk in cloud may in cloud multimedia streaming service. These make the risk management for multimedia streaming service in cloud computing is meaningful and imperative.

Business Model for Information Security (BMIS) is a widely recognized and available model published by ISACA (Information Systems Audit and Control Association). This is a generalized and dynamic security model which stands on a business level. To apply this model to cloud computing, we proposed a leveraged BMIS model by analysis of practical cloud risk causal factors in our previous research [9]. But this is a general cloud risk control model; for multimedia streaming service area, there must be some new characters need to be taken into consideration. This paper direct at cloud multimedia streaming service area, explore the new feature in this environment, try to apply the leveraged BMIS model to it and find an approach for risk management in this environment. 
The structure of the remainder of this paper is as follows. Section 2 introduces the related work of this paper. This section describes the situation of cloud based multimedia streaming service and the previous related work. Section 3, which is the core part of this paper, gets under way of model building. It includes three steps: first find out the new requirement in multimedia streaming service, then explore the association between the new requirement and the initial BMIS model, finally mapping the leverage point to new requirement for model implementation. Section 4 makes the conclusions of this paper, describes the advantage and meaning of the whole work, also point out the shortcomings of this paper.

\section{Related work.}

\subsection{Present development of multimedia broadcasting and existing problems}

In multimedia broadcasting, from DVB (Digital Video Broadcasting) to DMB (Digital Multimedia Broadcasting) or MBMS (Multimedia Broađcast Multicas seryice), the digital data transmission technology is nowadays in a rapid development. DVB, for example, has already experienced two generations. The first generation brought the specifications of DVBS/T/C Digital Video Broadcasting- Satellite / Terrestrial/Cable) and we got the standards of DVB-S2, DVB-T2, DVB-C2 in the second geperation. Nowadays, the DVB-HDTV, DVB3DTV, especially plus with GEM (Globall Executable Mild dleware) [1], broadcasting is closing to connection with internet little by little. Take advantage of the Java Micro Edition platform and specific APIs, GEM based DVB can realize four aspects: Broadcast, Packaged Media, IPTV, and Over-The-Top (OT) $)$, each of them has an inevitable connection with internet transmission (See Figure 1).

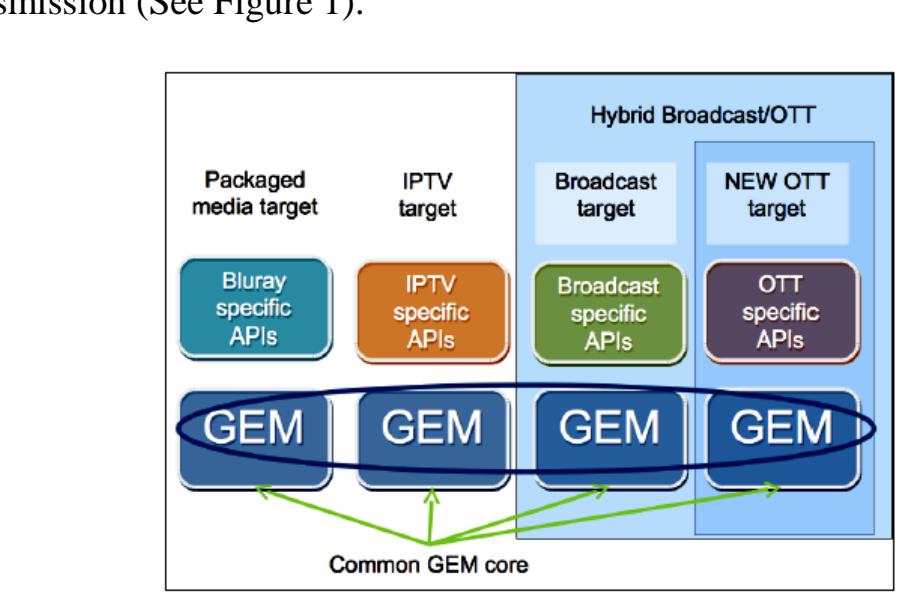

\section{Figure 1. The structure of GEM target specifications [1]}

The digital data transmission technology has mushroomed with the birth of DVB broadcasting. From the initial MPEG-1(like mp3 format) to MPEG-2 (widely used in Cable TV) and MPEG-4 (widely used in internet TV) and the recent many kinds of adaptive streaming, such as Apple's HLS (HTTP Live Streaming), Microsoft's Smooth Streaming, Adobe's HDS (HTTP Dynamic Streaming), MPEG-DASH (Dynamic Adaptive Streaming over HTTP), media contents gained a more and more higher quality transmission during the streaming technology development. During the broadcast, the data transmitted by a form named streaming. Here the streaming is a compression and download method which splits a traditional file into many blocks and can be played during these blocks are downloading through the internet. 
MPEG-DASH, for instance, is a recent hottest topic of streaming technology in multimedia broadcasting. It so-called can unifying the adaptive streaming world which can provide the highest quality of video transmission according to the bandwidth variation at any moment. Besides, it also furnishes a series of functions such as Time-shift viewing [2], Ads insertion [2, 3], Clock-drift control for live streaming [3,4] and so on. Since transmitted through internet, it also supports a common encryption with multi-DRM system for contents protection. Where is an internet, there always goes with risk and security problems, contents protection should be indisputably take consideration in broadcast streaming service.

\subsection{Cloud computing in television and broadcasting industry}

Traditional TV broadcasters need to own their infrastructure for contents collection, editing and processing in suitable formats etc. which need to take big investment in manpower and material resources. What's more, it needs constant update and maintenance which will take more costs. Cloud computing suits the remedy to the case.

Since the multimedia streaming service (like IPTV, OTT) always interact with the Internet, thus make it possible for utilization of cloud compuing in multimedia broadcasting industry.

Figure 2 depicts the fusion of traditional TV and cloud based streaming service broadcasting system. Traditionally, the contents providers need to edit and compress the programs by their own installations and thendistribute to customers through the basic DVB network; through the cloud streaming service, use the cloud software to do graphics and compression, media contents can transmit to both dable TV customers and PC, Mobile devices.

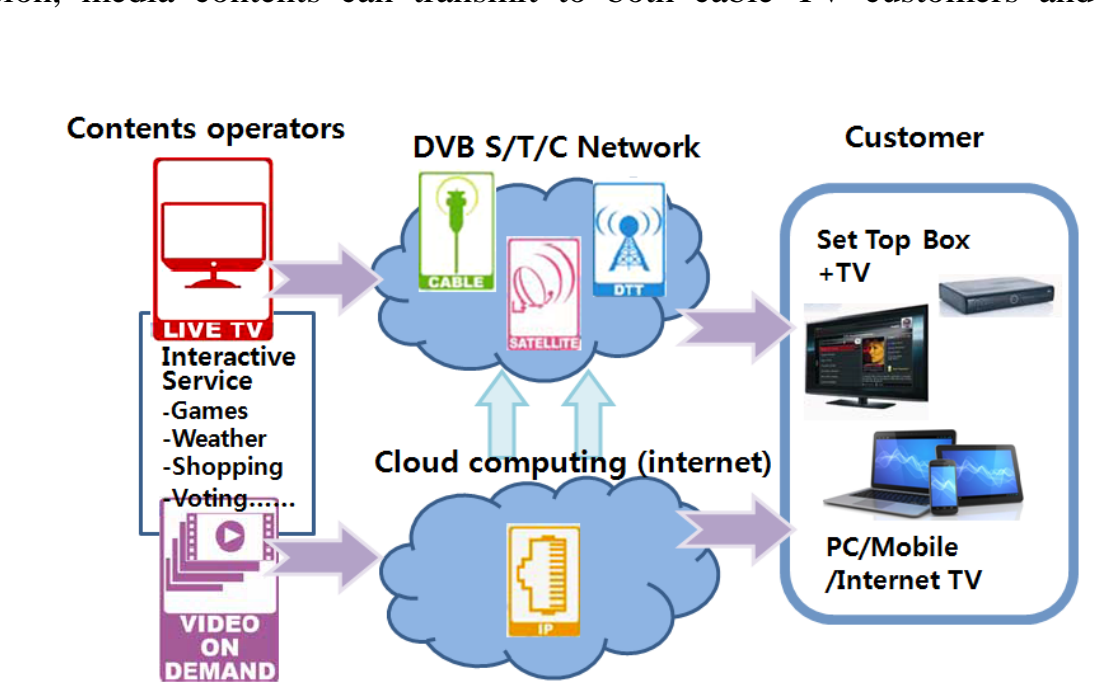

\section{Figure 2. Fusion system of traditional TV and cloud based streaming service}

Cloud computing is well known with three kinds of service: SaaS (Software as a service), PaaS (Platform as a service), IaaS (Infrastructure as a service). The same thing can be bring into broadcast streaming service. For multimedia broadcasters, SaaS cloud can offer virtual desktop, graphics; PaaS cloud can provide OS (Operating system), database, development tool and web server; IaaS cloud can supply virtual machine, storage, metadata backup, etc. [5].

Now a lot of enterprises have already moved to cloud based broadcasting service. Axis World Graphics, for example, one of the first entrants into the cloud based broadcast area which provides contents creation software and custom graphic [5]; Qtube supply browser, edit and transformer to users or content creators and ensure they can interact with, edit, or view their contents [6]. In China, PaaS cloud also has been put into agenda. The CCTV 
(China Central Television Station) stated this already developed two platform for cloud computing; the news cloud platform and content service cloud platform, and pointed that cloud computing will be the core technology in tri-network integration and China's NGB (Next Generation of Broadcasting television network) plan [7].

Cloud based streaming service saves troubles of maintenance also reduces the production costs for broadcasters and supply a high quality image transmission to their customers, but the contents management will be an arduous task. Cloud itself breeds the potential risk and security problems.

\subsection{BMIS and cloud applied BMIS model}

The original BMIS model is a property belongs to ISACA (Information Systems Audit and Control Association) and the full name of BMIS is the Business Model for (nformation Security [8]. It's a three-dimensional, pyramid-shaped model and there are four elements (organization, people, process, and technology) and six dynamic interconnections (governing, culture, enabling and support, emergence, human factors and architecture), in the construction of this model (See it in Figure 3).

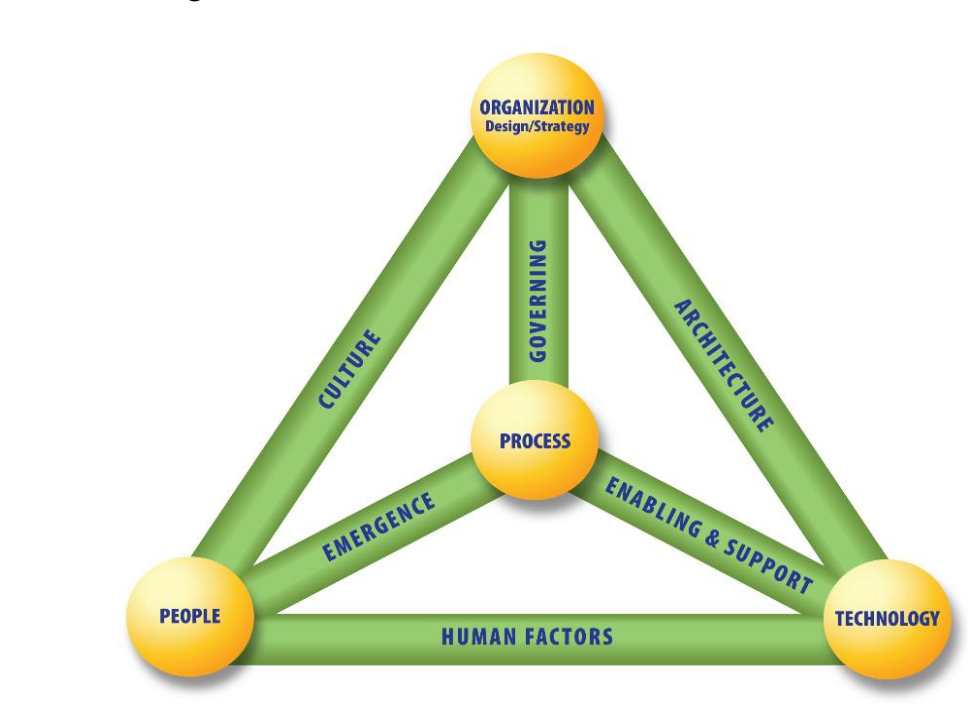

Figure 3. Business Model for Information Security

BMIS model aims to recognize and accommodate dynamic relationships both internal and external to the business. Cloud computing service can be counted as a third-party service in essence, thus it's more probable under threats of both internal and external aspects. BMIS acts appropriately to the situation, according to ISACA, BMIS is a predictive and proactive model, not just concerning traditional information security but including privacy, linkage to risk, physical security and compliance [8]. Furthermore, BMIS model is labeled as the combination of information security program and business goals, which right match the ultimate purpose of enterprise operation: benefit and effectiveness.

To apply to cloud environment, through analysis [9], we found the essentially causal risk factors for each kind of cloud service problem, (See it in table1) and mapped them into BMIS's four elements respectively (See it in Table 2). To enhance the effectiveness of this model, we developed it use system leverage points and proposed a leveraged BMIS model in the previous research. The leveraged cloud applied BMIS model ensure the risk control directly focus on the realistic problems in cloud computing and the leverage points supply a 
management mode to optimize the resource of cloud related enterprise. Cloud based streaming service, as one kind of cloud service can also under administration of this model.

Table 1. Practical cloud risk type and their casual factors

\begin{tabular}{|c|c|c|c|c|}
\hline Technology & Trust & Data & Compliance & Measurability \\
\hline $\begin{array}{l}\text { 1. Encryption } \\
\text { 2. VM technology } \\
\text { 3. Identification } \\
\text { 4. Authentication } \\
\text { 5. Authorization }\end{array}$ & $\begin{array}{l}\text { 6. Staff reliability } \\
\text { 7. Human resources } \\
\text { 8. Service model } \\
\text { 9. Outsourcing level } \\
\text { 10. Scale \& Structure } \\
\text { 11. Fault control } \\
\text { 12. Feedback loops }\end{array}$ & $\begin{array}{l}\text { 13. Data update } \\
\text { 14. Physical loss } \\
\text { 15. Disaster } \\
\text { recovery } \\
\text { 16. Business } \\
\text { continuity }\end{array}$ & $\begin{array}{l}\text { 17. Contract (SLA) } \\
\text { 18. Internal security } \\
\text { policy \& Regulation } \\
\text { 19. External standards } \\
\text { \& laws }\end{array}$ & $\begin{array}{l}\text { 20. Auditing } \\
\text { (business; } \\
\text { data traffic; } \\
\text { security) }\end{array}$ \\
\hline
\end{tabular}

The concept of leverage is one of the most powerful in all of science. Archimedes proved the Law of the Lever and applied the law in a variety of inventions. Historian John Tzetzes, writing in the 12th century, wrote that Archimedes said "Give me a place to stand and with a lever I will move the world" [10]. In systems thinking a leverage point is a place in a system's structure where a solution element can be applred. It's a low leverage point if a small amount of change force causes a small change in system behavior. It's a high leverage point if a small amount of change force causes a large change in system behavior [11].

An article named "Leverage points to intervene in a system" was published in 1999 by Donella Meadows [12]. Meadøws, who worked in the field of systems analysis, proposed a scale of places to intervene in a system. Awareness and manipulation of these levers is an aspect of self-organization and can lead to oollective intelligence. In this article, there are twelve leverage pointslisted as below:

(1) Power to transcend paradignts

(2) Mindset or paradigm out of which the system arises

(3) The Goal of the system

(4) Power to add, change, evolve, or self-organize system structure

(5) Rules of the system (such as incentives, punishment, constraints)

(6) Structure of information flow

Table 2. Casual risk factors mapping with each BMIS element

\begin{tabular}{l|c|c}
\hline \multirow{4}{*}{ EMIS } & Elements & Subordinate CSFs \\
\cline { 3 - 3 } Model & \multirow{3}{*}{$\begin{array}{c}\text { Organization } \\
\text { (design/strategy) }\end{array}$} & 1. Business continuity \\
\cline { 3 - 3 } & & 2. Contract (SLA) \\
\cline { 3 - 3 } & & 3. Internal security policy \& Regulation \\
\cline { 3 - 3 } & & 4. Outsourcing level \\
\cline { 3 - 3 } & & 6. Sault control \\
\cline { 3 - 3 } & & 7. Scale \& Structure \\
\hline
\end{tabular}




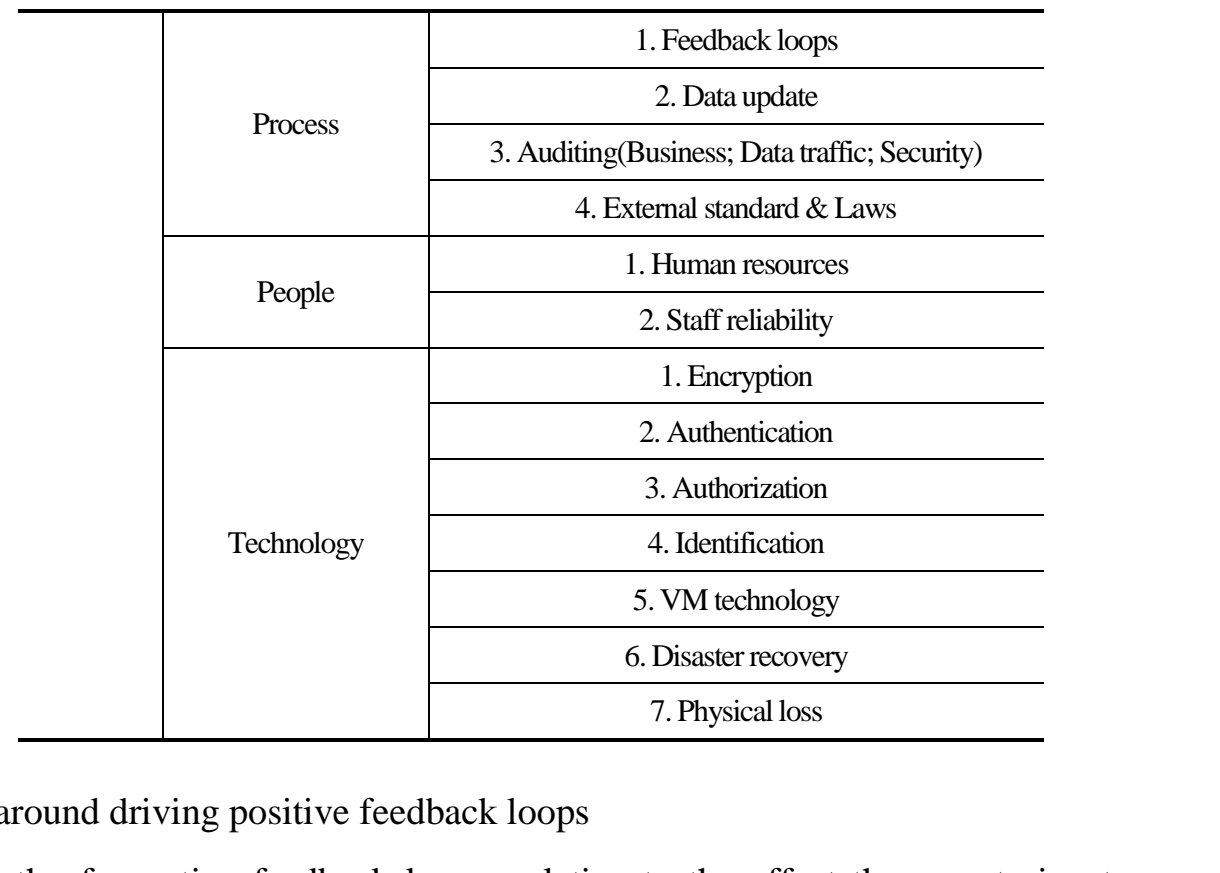

(7) Gain around driving positive feedback loops

(8) Strength of negative feedback loops, relative to the effect they are trying to correct against

(9) Length of delays, relative tô the rate of system ehanges

(10) Structure of material stocks and flows lsuch as transport network, population age structures)

(11) The size of buffers and other stabilizing stocks, relative to their flows

(12) Constants, parameters, nûmbers (such as subsidies, taxes, standards)

The twelve leverage points listed in order of system components' importance. Compared with BMIS model, it also mentioned some elements: structure, organization, human factor in detail, and gives an ordinary sequence for these elements which you should pay attention in a system. It's sure that there is a leverage point in every system; we can make the resource optimization by mapping with leverage points. This twelve leverage points lead a resource integration concept, and can be helpful to optimize the resource distribution. In the light of resource optimižation, leverage points means getting the maximum output with a minimum input the management model can be more benefited through the leverage point mapping process.

\section{Model Building}

\subsection{New requirement exploration in cloud based multimedia streaming risk management}

To control the potential risk in cloud based multimedia streaming service, compared with general cloud service; we first need to excavate the different requirements in multimedia streaming area.

Practically, to begin with, there is different user scope in multimedia streaming service. General cloud service always more tend to specific group or certain people. For example, 
cloud ERP designed for business users in most case; cloud healthcare service focus on patients; cloud gaming always serve for gamers; much less, PaaS and IaaS oriented cloud are much more tend to professionals to use. However, there is no specific or certain group for multimedia streaming service; everyone can be an audience of streaming broadcast. Cloud based streaming service need to serve more general people which require a more humanized and simplified UI (user interface) design. In the second place, multimedia streaming service is more value on quality of service (QoS). It's because streaming service itself is labeled as it can supply more interaction and higher image quality to users compared with traditional TV broadcasting. Not just it can be use or not, but whether it can provide a smooth, continuous and high definition streaming is very important to users. This requires a good user experjence which called UX in service aspect. In another word, unworkable user interface and unstable or inferior image transmission will bring a risk in multimedia streaming service. Both UX and UI problems are eventually attributed to one aspect: performance [13, 14].

Academically, Kent pointed that the relative performance importance is different between connection-oriented and broadcast environment. The system performance must be taken into account when designing protocols for secure communication [15].

Through above analysis, performance undoubtedly) is a important role in cloud based streaming service risk management.

Detailedly, performance is a requirement or atárget in ctoud based streaming risk control process. It can involve three aspects in streaming service problems.

(1) A workable UI: Not just the deliberate Sabotage but also the feasibility of the system itself bring a risk in service operation. This aspect ean be affected by technology support and human resource. More sufficiemtechnology support and human resource will ensure a more interactive and humanized user interface.

(2) A good UX: Even if there is no external hostile aggression, the breakage physical network or since the problems from protocols and various kinds of firewall, users may be subjected to video frequency interrupt risk such as $4 \mathrm{XX}$ client error or $5 \mathrm{XX}$ server error or repeated $3 \mathrm{XX}$ redirection message 116]. This aspect refers to user feedback message and process control, constant and real-time monitoring can help to discover the problem in time and ensure a smooth multimedia streaming service for users.

(3) SLA requirement. The word performance itself, for cloud based streaming service, means it should provides a high quality, high resolution image and high bandwidth service to users as much as possible. This aspect refers to the contract quality problem, service level agreement (SLA) should responsible for the performance in contract level [17]. A good and clear performance clause will reduces the unnecessary contractual dispute risk.

\subsection{The connection between the new requirement and cloud applied BMIS model}

In previous work, we proposed a cloud applied leveraged BMIS model to make an effective risk management in cloud environment. It operates like figure 4, each element take charge of several cloud risk causal factors, and each factor is assigned one appropriate leverage point to optimize the resource allocation. 


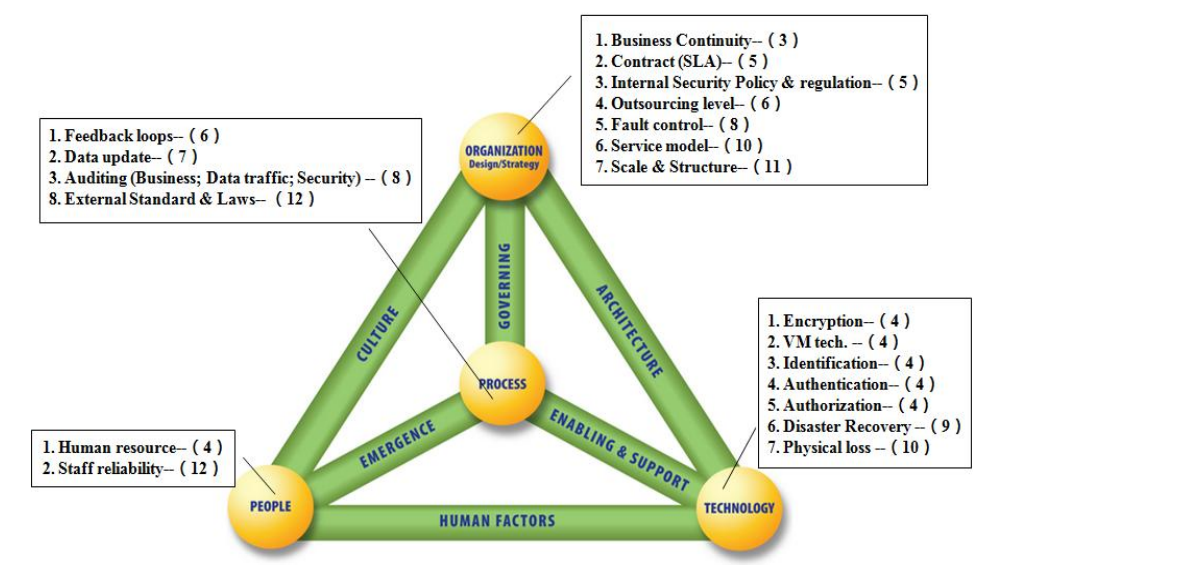

Figure 4. Cloud applied leveraged BMIS moder [9]]

The proposed risk management model supplies an integrated risk control function to general cloud service. Although the basis risk control for Cloud broadcast service should comply with the general cloud risk control, directysing of this model to the specific cloud multimedia streaming service area will beosomeynat thoughtiess. Through above analysis, we found the new requirement in cloud broadcast environment. the performance requirement is requisite for risk management in cloud multimedia streanting service. To apply this leveraged BMIS model to this area, we need 10 find out the cornection between the new requirement and the proposed cloud applied BMIS model.

As mentioned above, performance requirement refers to three aspects.

First, the UI aspect, it can Involve technology and people elements in initial BMIS model. For technology element it needs a "UI design" technique support. This factor do not exist in original leveraged BMIS model, it's a new factor which we need to take into consideration in broadcast environment For people element, it relates to "human resource" factor.

Second, the UX aspect, it can connect with process element. For detail level, it relates to the specific factor feedback loops"

Third, the SLA requirement, it can connect with organization element. For detail level, it relates to the specific factor "contract (SLA)".

Thus the performance requirement involves all elements existed in BMIS model. Organization people, process and technology, each element holds a causal factor which can correspond with the performance requirement. The new relationship built as follows. (Figure 5 below)

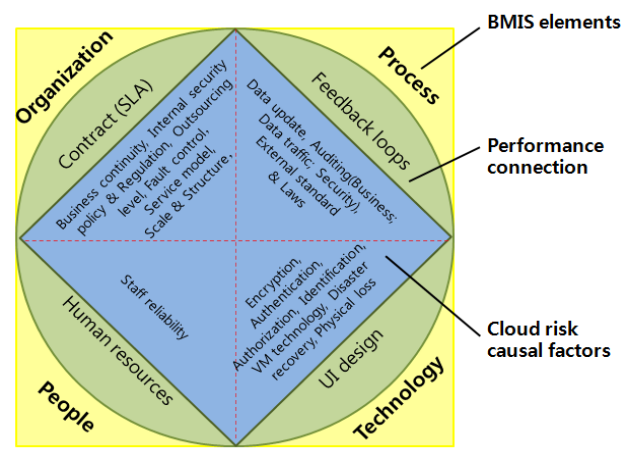

Figure 5. Performance connection with cloud applied BMIS model 
Figure 5 illustrates the performance connection with cloud applied BMIS model the innermost foursquare represents the specific risk causal factors which under control of certain element; the intermediate circle represents the overlap factors which correspond with the performance requirement; the outermost foursquare on behalf of the four elements which in charge of all cloud risk causal factors.

From the figure, it's easy to see that the performance definitely not an individual factor but a connector for the holistic system. It's worth noting that, performance here is a requirement which can associate all elements in BMIS model; it also can be seen as one of risk management target (although the ultimate target is for business benefit and continuity) in cloud multimedia streaming service environment.

\subsection{Cloud multimedia streaming service applied leveraged BMIS model}

Since performance as a new target and requirement added into the BMIS model for cloud multimedia streaming risk management, we aim to find the new item sleverage point for enhancing the effectiveness of this model.

In order to mapping performance with leverage point, first we need a better understanding of the meaning of every leverage point. We read the "twelve leverage points" in-depth and conclude the key conception of every leverage point as below. (See Table 3)

According to the concept description of performance requirement and the keyword description of each leverage point, performance is more close to leverage point 3 . That's because performance as a risk management target and a connecter of all model elements (involves the physical maintenance, teedback loops ete.) helps to improve the service quality and ensure the customer reterition. This right matches with leverage point 3 which also mentioned a system goal and increase of marketshare.

Table 3. The keyword description of twelve leverage points

\begin{tabular}{|c|c|}
\hline Leverage points & Concept and keyword Description \\
\hline & $\begin{array}{l}\text { Stay flexible } \\
\text { - Keep oneself unattached in a paradigm } \\
\text { - No paradigm, you can choose whatever one will help to achieve } \\
\text { your purpose. }\end{array}$ \\
\hline $\begin{array}{l}\text { 2. Mindset or paradign out of } \\
\text { which the system arises }\end{array}$ & $\begin{array}{l}\text { Paradigm change } \\
\text { - Keep pointing at failures in old paradigm } \\
\text { - Keep speaking louder assurance from the new one } \\
\text { - Insert people with new paradigm in place of public visibility and } \\
\text { power. }\end{array}$ \\
\hline l of the system & $\begin{array}{l}\text { Whole system goal (physical, stocks, flows, feedback loops, } \\
\text { information flows, self-organizing behavior, will be twisted to } \\
\text { conform to that goal.) } \\
\text { To grow, to increase market share, to bring the world more and } \\
\text { more under the control of corporation. }\end{array}$ \\
\hline $\begin{array}{l}\text { 4. Power to add, change, evolve, or } \\
\text { self-organize system structure }\end{array}$ & $\begin{array}{l}\text { System education } \\
\text { - Adding completely new physical structures, such as brains or } \\
\text { wings or computers. } \\
\text { - Adding new negative or positive loops } \\
\text { - Making new rules }\end{array}$ \\
\hline 5. Rules of the system & $\begin{array}{l}\text { - The rules of the system define its scope, its boundaries, its degree } \\
\text { of freedom } \\
\text { - Contracts are need to be honored } \\
\text { - A system with rules designed by corporations, run by corporations } \\
\text { for the benefit of corporations }\end{array}$ \\
\hline
\end{tabular}




\begin{tabular}{|c|c|}
\hline 6. Structure of information flow & $\begin{array}{l}\text { - Information feedback } \\
\text { - A comprehensive, timely market information flow delivering } \\
\text { information to a place where it wasn't going before and therefore } \\
\text { causing people to behave differently }\end{array}$ \\
\hline $\begin{array}{l}\text { 7. The Gain around driving positive } \\
\text { feedback loops }\end{array}$ & $\begin{array}{l}\text { Self-reinforcing } \\
\text { - The more it works, the more it gains power to work some more } \\
\text { - Controlling of growth rate }\end{array}$ \\
\hline $\begin{array}{l}\text { 8. Strength of negative feedback } \\
\text { loops, relative to the effect they } \\
\text { are trying to correct against }\end{array}$ & $\begin{array}{l}\text { Self-correcting (Information and control part) } \\
\text { - Keep the appointed stock at or near its goal } \\
\text { - The accuracy and rapidity of monitoring } \\
\text { - The quickness and power of response }\end{array}$ \\
\hline $\begin{array}{l}\text { 9. Length of delays, relative to the rate of } \\
\text { system changes }\end{array}$ & $\begin{array}{l}\text { Timely information, timely response } \\
\text { - Delay, too short cause overreaction, too long cause damped } \\
\text { - Its critical relative to rates of change in the system state that the } \\
\text { feedback loop is trying to control }\end{array}$ \\
\hline 10. Structure of material stocks and flows & $\begin{array}{l}\text { - The plumbing structure, the stock and } \\
\text { arrangement } \\
\text { - Physical building/structure }\end{array}$ \\
\hline $\begin{array}{l}\text { 11. The size of buffers and other } \\
\text { stabilizing stocks, relative to } \\
\text { their flows }\end{array}$ & $\begin{array}{l}\text { - The stabilizing power } \\
\text { - Moderate buffer } \\
\text { - Constantinventory to face the occasional fluctuations }\end{array}$ \\
\hline $\begin{array}{l}\text { 12. Constants, parameters, number (such as } \\
\text { subsidies, taxes, standards) }\end{array}$ & 6r parameters \\
\hline
\end{tabular}

In the previous research, we alreddy mapped all cloud risk causal risks with leverage pints, integrate with performance target, the holistic risk management model for specific cloud multimedia streaming service environment as follows: (See Figure 6)

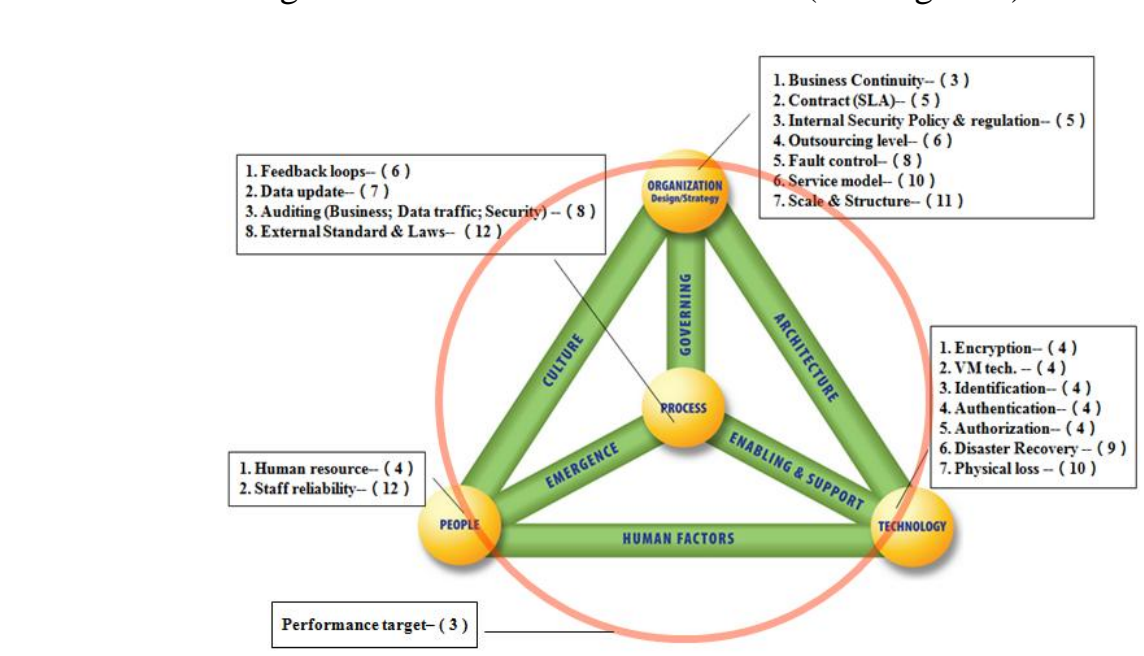

\section{Figure 6.The cloud multimedia streaming service applied risk management model}

The above figure shows a leveraged risk controlling model for multimedia streaming service in cloud computing. Based on the initial BMIS model and the cloud applied leveraged remodeling, with considering of the specific character in multimedia streaming service, we got a performance targeted leveraged BMIS model. 
This is a hollow sphere model which involves performance requirement and all cloud risk causal factors in it. Compared with the initial BMIS' "pairwise correlation", the performance spherical shell at outermost layer associate all four elements to ensure the orientation of risk control. In the spherical shell, as the second layer, four elements supervise the different aspects of the management system, and the element also parewise connected with each other. Inside the four elements, as the third layer, all sorts of cloud risk causal factors take charge of micro-control in detail. Cloud multimedia streaming service as a cloud based service, the basic risk and problems can be controlled by internal factors of each element, the external performance target helps to improve the effectiveness of model operation.

\section{Conclusion}

This paper proposes an approach of risk management for multimedia/streaming service in cloud computing. In cloud service industry, the orisk always points to bidirection, both cloud service providers and cloud service clients can be subjected to damage. Traditional security models tend to corporate, in house environment, regard outsourcing or third party as a business issue that only addfess at contractual or legal level. The initial BMIS model is an interactive and dynamic model, which can act on both internal and external sides. Based on the cloud applied leveraged BMIS model, we considerate the new feature required in myltimedia streaming service, remodel and adjust this model to cloud streaming area. Cloud based broadcasters and relevant enterprises can use this model for a performance targeted effective risk management.

Although this model takes the spedific consideration on multimedia streaming service, leverage the resource allocation, aim to effectuate the risk management, but the lack of model verification is still an insufficiency for this/paper. Quantitative decision-making method can be helpful for model verification and validation.

In the further work, we will try to use quantitative method such as AHP to enhance the utilization rate of this mødel and strive to get a more precisel approach for cloud based multimedia streaming tisk management.

\section{ACKNOWLEDGEMENTS}

This research was supported by Basic Science Research Program through the National Research Foundation of Korear (NRF) funded by the Ministry of Education (2013R1A1A2011581), and was supported by the Donggák University Research Fund of 2013.

\section{References}

[1] S. Siebert, "DVB: Developing Global Television Standards for Today and Tomorrow", Technical Symposium at ITU Telecom World (ITU WT), (2011) October 24-27; Geneva.

[2] T. Stockhammer, "Dynamic Adaptive Streaming over HTTP - Design Principles and Standards", In Proceedings of the second annual ACM conference on Multimedia systems (MMSys11), (2011) New York, USA.

[3] A. Vetro, "The MEEG-DASH Standard for Multimedia Streaming Over the Internet", (2011), October December, pp. 62-67.

[4] A. Salo (RGB Networks), MPEG DASH: A Technical Deep Dive and Look at What's Next, (2012).

[5] R. Prakash, S. Lamdharia and D. G. Chandra, "Scope of Cloud Computing in Television Broadcasting Industry: A Study", International Conference on Communication Systems and Network Technologies (CSNT), (2013), pp. 371-375.

[6] Quantel Whitepaper, QTube Global Media Workflow, Revision 2.7, (2011) October 13.

[7] N. We and P. Xu, "Cloud Computing and its Application in Television and Broadcasting Industry", IEEE3rd International Conference on Software Engineering and Service Science (ICSESS), (2012), pp. 372-375.

[8] ISACA, "An Introduction to the Business Model for Information Security", (2010). 
[9] Y. S. Pang and Y. J. Song, "The leveraged BMIS Model for Cloud Risk Control”, JIPS, vol. 11, (2013) (to be published).

[10] http://en.wikipedia.org/wiki/Lever\#cite_note-1.

[11] S. Sembhi, "The Business Model for Information Security", RSA Conference, (2010).

[12] D. Meadows, "Leverage Points: Places to Intervene in a System", The Sustainability Institute, (1999).

[13] http://en.wikipedia.org/wiki/User_experience.

[14] http://en.wikipedia.org/wiki/User_interface.

[15] S. T. Kent, "Security Requirements and Protocols for a Broadcast Scenario", IEEE Transactions on Communications, vol. 29, no. 6, (1981), pp. 778-782.

[16] ISO/IEC23009-1, Information technology - Dynamic adaptive streaming over HTTP (DASH), (2012).

[17] G. Lennon, "Cloud computing for broadcasters", Broadcast Engineering, (2011) June 1.

\section{Authors}

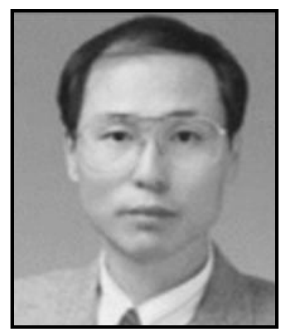

\section{YouJin Song}

He received a Ph.D. degree in Anformation Security from Tokyo Institute of Technology, Japan in 1995. He has been a professor at Dongguk University since 1996. His research interests include privacy protection, secret sharing, cloud security and its application, multimedia security.

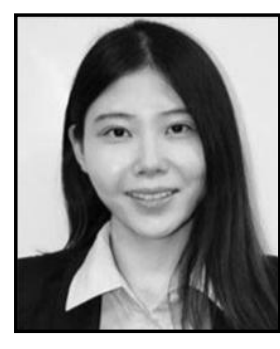

\section{Yasheng Pang}

She received a master degree in Electronic Commerce Technology from Dongguk University, Korea in 2013. Her research interests include cloâd security, risk management, data mining and cloud broadcasting. 